\title{
VENEZUELA: PETRÓLEO Y POLÍTICA
}

\section{DEPENDENCIA DE LA RENTA PETROLERA Y POTENCIAL EXPORTADOR PARA LA DIVERSIFICACIÓN ECONÓMICA DE VENEZUELA}

\author{
Kleidy Y. Espinoza M.
}

Aspiranta a Ph.D. (kleidyes@gmail.com)

Departamento de Economía Mundial y Negocios Internacionales

\section{Tatiana V. Sidorenko}

Ph.D. (Economia),prof. (tsidoren@yandex.ru)

Departamento de Economía Mundial y Negocios Internacionales

Universidad de Finanzas adjunta al Gobierno de la Federación de Rusia Leningradskiy prospekt, 49, Moscú,125993, Federación de Rusia

Recibido el 25 de agosto de 2020

Aceptado el 10 de diciembre de 2020

DOI: $10.37656 / \mathrm{s} 20768400-2021-1-04$

Resumen. El artículo se basa en el análisis de la cultura rentística que ha tenido Venezuela de la monoexportación de materias primas y específicamente del petróleo. Situación que la ha mantenido en una posición de alta vulnerabilidad debido a que el mercado exportador del país está basado en un $83 \%$ en la venta del petróleo y sus ingresos externos están sujetos a la volatilidad de los precios del mercado financiero internacional. Aunado a la precaria situación económica, política y social debido a factores externos e internos que han sumergido al país suramericano en el peor colapso de toda su historia. Desde hace 60 años y hasta la actualidad se han diseñado políticas para el comercio exterior del país, con el fin de diversificar la producción nacional, y reducir la dependencia de la renta petrolera, pero ninguna ha sido suficientemente eficaz para lograr la expansión y desarrollo del aparato productivo nacional. Venezuela posee un fuerte potencial en cuanto a recursos naturales que favorecen la diversificación, pero para ello se requiere inversión y financiamiento internacional, desarrollo tecnológico y de infraestructura para así levantar al pais de la profunda crisis en la cual se encuentra sumergida. Metodológicamente nuestra investigación es de carácter histórico analítico, 
Dependencia de la renta petrolera y potencial exportador para la diversificación económica de Venezuela que, usando datos cualitativos y estadística descriptiva, busca comprender los fenómenos señalados.

Palabras clave: Venezuela, crisis económica, dependencia, diversificación, exportaciones, importaciones, productos no tradicionales, renta petrolera

\title{
OIL INCOME DEPENDENCY AND EXPORT POTENTIAL FOR ECONOMIC DIVERSIFICATION OF VENEZUELA.
}

\author{
Kleidy Y. Espinoza M.
}

Ph.D. student (Economics), (kleidyes@gmail.com)

Department of World Economy and International Business

\section{Tatiana V. Sidorenko}

Ph.D. (Economics), associate prof. (tsidoren@yandex.ru)

Department of World Economy and International Business

Financial University under the Government of the Russian Federation 49, Leningradskiy Prospekt, Moscow, 125993, Russian Federation

Received on August 25, 2020

Accepted on December 10, 2020

DOI: $10.37656 / \mathrm{s} 20768400-2021-1-04$

Abstract. The article is based on the analysis of the rentier culture that Venezuela has had of the mono-export of raw materials and specifically of oil. Situation that has kept it in a highly vulnerable position due to the fact that the country's export market is based $83 \%$ on the sale of oil and its external income is subject to the volatility of prices in the international financial market. Coupled with the precarious economic, political and social situation, they have plunged the South American country into the worst collapse in its entire history. For 60 years and up to the present, policies have been designed for the country's foreign trade, in order to diversify national production, and reduce dependence on oil income, but none have been effective enough to achieve the expansion and development of the apparatus national productive. Venezuela has strong potential of natural resources that favor diversification, but this requires investment and international financing, technological development and infrastructure to lift the country out of the deep crisis in which it is submerged. Methodologically, our research is of an analytical historical nature, which, using qualitative 
Kleidy Y. Espinoza M., Tatiana V. Sidorenko

data and descriptive statistics, seeks to understand the aforementioned phenomena.

Keywords: Venezuela, economic crisis, dependence, diversification, exports, imports, non-traditional products, oil income

\title{
ЗАВИСИМОСТЬ ОТ НЕФТЯНЫХ ДОХОДОВ И ЭКСПОРТНЫЙ ПОТЕНЦИАЛ ДЛЯ ДИВЕРСИФИКАЦИИ ВЕНЕСУЭЛЬСКОЙЭКОНОМИКИ
}

\author{
Клейди И. Эспиноса М. \\ Аспирантка (Экономика), (kleidyes@gmail.com) \\ Департамент мировой экономики и международного бизнеса \\ Татьяна Викторовна Сидоренко \\ Канд. экон. наук, доиент BAK (tsidoren@yandex.ru) \\ Департамент мировой экономики и международного бизнеса \\ Финансовый университет при Правительстве РФ \\ РФ, 125993, Москва, Ленинградский проспект, 49 \\ Статья получена 25 августа 2020 г. \\ Статья принята 10 декабря 2020 г.
}

DOI: $10.37656 / \mathrm{s} 20768400-2021-1-04$

Аннотация. Статья базируется на анализе культуры рантье, которая сложилась в Венесуэле, специализирующейся в течение долгого времени преимущественно на экспорте сырья и, в частности, нефти. Данная ситуация предопределяет высокую степень уязвимости странь поскольку 83\% ее экспорта составляет продажа нефти, а доходы от экспорта зависят от волатильности иен на международном финансовом рынке. К этому добавляется нестабильная экономическая, политическая и сочиальная ситуаџия, вызванная внешними и внутренними факторами, которые погрузили экономику этой южноамериканской страны в наихудшее положение за всю ее историю. В течение последних 60 лет в Венесуэле претворялась в жизнь внешнеторговая политика, направленная на диверсификацию начионального производства с иелью снижения зависимости от получаемых от экспорта нефти доходов. Однако она оказалась недостаточно эффективной для развития национального 
Dependencia de la renta petrolera y potencial exportador para la diversificación económica de Venezuela производственного аппарата. Для выхода страны из кризиса также требуется приток иностранных инвестиций, содействие научнотехническому развитию и создание современной инфраструктуры.

Ключевые слова: Венесуэла, экономический кризис, зависимость, диверсификаиия, экспорт, импорт, нетрадиционные товары, нефтяная рента

\section{Algunos aspectos de la Venezuela agrícola y petrolera}

Venezuela se caracterizó por la existencia de una fuerte tradición agrícola, los campos se encontraban altamente poblados; pero una vez que comenzó el auge petrolero, gran parte de esa población campesina abandonó los campos y migró a las ciudades. Se producen cambios profundos en la dinámica económica, social y política del país. Venezuela deja de ser un país agroproductor y agroexportador para convertirse en un predominantemente monoproductor y monoexportador de petróleo. Sin embargo, desde los tiempos de la colonización española ha estado arraigada la cultura rentista, debido ala dependencia de la economía de un rubro en particular, como lo fue, en primer lugar, el cacao en la segunda mitad del siglo XVII y primera mitad del siglo XVIII. La exportación del cacao convertía al país en agroexportador dependiente del monocultivo, al que correspondía el $75 \%$ del total de exportaciones. Posteriormente fue desplazado por el café bajo el mismo sistema y en el siglo XIX, representando el $83 \%$ de las exportaciones, situación que continuó hasta la aparición del petróleo, el cual ha llegado a representar incluso el 95\% de éstas.

Venezuela se fue convirtiendo en un país destinado a la importación de bienes y servicios, antes que a la producción.

A partir de la década de los 50 del siglo pasado, Venezuela alcanzó las más altas tasas de crecimiento industrial. Se 
materializó un importante y sostenido crecimiento del PIB manufacturero, y continuó el crecimiento hasta el año 1978 [1].

Los sectores petroquímico y siderúrgico fueron los de mayor relevancia, los cuales tenían importantes ventajas comparativas y fueron de alto nivel estratégico. El sector manufacturero, desde 1955 a 1959, presentó tasas de crecimiento elevadas, especialmente las industrias intermedias y las industrias mecánicas, que crecieron a un ritmo de $20,8 \%$ anual, y con un incremento del PIB de 9,3\% [2].

Enlas décadas de los 60 y 70 luego de la aplicación de políticas proteccionistas e iniciativas para promover las exportaciones no petroleras, se impulsó el modelo de sustitución de importaciones y el producto industrial se elevó en 9,1\%, destacando: industrias básicas, hierro y acero: $88,7 \%$, materiales de transporte: $15,7 \%$, los textiles: 13,2\%, alimentos: 9,1\%, tabaco: $6,7 \%$, y prendas de vestir: $5,3 \%$. Mientras que la agricultura en la década de los 70 tuvo crecimiento a un ritmo de $5,8 \%$ anual, uno de los crecimientos agrícolas más altos del mundo [3].

Cabe destacar que posterior a las políticas mencionadas Venezuela se incorpora al acuerdo subregional Grupo Andino en 1973, promoviendo de esta manera la liberalización comercial a nivel regional; en el bloque se registró durante variosaños una balanza comercial superavitaria con países como Colombia, debido precisamente a la exportación de bienes no tradicionales con alto valor agregado y basada en incentivos fiscales. En este sentido el producto manufacturero creció al 7,9\% promedio anual para pasar del último al primer lugar en términos de su producto industrial entre los países medianos de América Latina (Colombia, Chile y Perú). 
Dependencia de la renta petrolera y potencial exportador para la diversificación económica de Venezuela

En América Latina durante el período 1920 - 1960, Venezuela fue considerada la economía más exitosa, la de mayor crecimiento económico hasta los años ochenta, sin embargo, también hubo un aumento de la brecha tecnológica,ausencia de encadenamientos $\mathrm{y}$, especialmente, una temprana y excesiva desindustrialización para los niveles de productividad.

Esta desindustrialización se debió en gran medida a lo que muchos economistas llaman como Enfermedad Holandesa, generada por los altos ingresos provenientes de la renta petrolera que influyó en el aumento de las importaciones de bienes y servicios, por la apreciación de la moneda nacional, el Bolívar, trayendo como consecuencia una reducción o paralización de la producción nacional debido a la disminución de los costos que representan las importaciones en comparación con la producción, causando a su vez una contracción de la producción agropecuaria, manufacturera y el deterioro de la competitividad de los bienes transables (exportables).

\section{Dependencia de la renta petrolera}

Venezuela tiene varios años sumergida en una profunda crisis económica y social que demuestra que tener las reservas probadas de petróleo más grande del mundo según la Organización de Países Exportadores de Petróleo (OPEP) no basta y no es suficiente para que un país pueda desarrollarse en todas las áreas y generar condiciones de vida favorables para sus ciudadanos si no hay políticas adecuadas en materia económica que además promuevan de una manera eficaz la diversificación del aparato productivo nacional y en consecuencia de las exportaciones, con el fin de contribuir al desarrollo sostenible de 
la nación para evitar estar expuestos a niveles tan altos de vulnerabilidad ante situaciones externas como las crisis financieras internacionales, caída de los precios del petróleo, sanciones y bloqueos económicos.

Con la actual crisis económica que atraviesa Venezuela, cobra vigencia la frase que fue inspirada por el intelectual venezolano Alberto Adriani (1898-1936), y que precisó posteriormente el escritor Arturo Uslar Pietri (1906-2001), "sembrar el petróleo", haciendo el enfoque respecto a la necesidad de que los recursos provenientes del petróleo se reinvirtieran para el desarrollo y fortalecimiento de la agricultura venezolana y la precisión de hacerla más competitiva, impulsando la economía nacional. Afirmó lo siguiente:

"Si en este momento, por azar infortunado del destino, los precios del petróleo bajaran de una manera importante en el mercado mundial, Venezuela sería un caso para la Cruz Roja Internacional”.

Antonio Ecarri, abogado venezolano y presidente de la Fundación Casa Arturo Uslar Pietri, señala que más que una profecía, la frase "sembrar el petróleo" se convirtió en la gran tarea inconclusa de los venezolanos.

Las divisas en promedio que ingresan a Venezuela por concepto de exportaciones desde el año 1950 en un95\%se deben a la renta petrolera, y en consecuencia la economía venezolana depende de los precios y los niveles de producción de este hidrocarburo. Característica que la ha ubicado en situación de extrema vulnerabilidad, ya que estos ingresos de divisas dependen de la volatilidad de los precios del crudo en el mercado financiero internacional.

El petróleo representa el $83 \%$ del mercado exportador de Venezuela, el $11 \%$ está representado por metales preciosos, el 
Dependencia de la renta petrolera y potencial exportador para la diversificación económica de Venezuela $2,5 \%$ por los químicos,el $2,1 \%$ por las exportaciones de metales y $1 \%$ suman las exportaciones de cacao y maíz.

Por su parte, durante el período 2012-2018 el ingreso en divisas por concepto de exportaciones petroleras pasó de US\$ 95.569 millones a US\$29.810 millones. Es decir, los ingresos en divisas cayeron en un $68,1 \%$ durante el período mencionado. Uno de los factores que incidió en esta caída fue la disminución en 45\% del precio del petróleo que pasó de 103,46 US\$ a 57,42 US\%, y, por otra parte, la producción de petróleo cayó de 2,7 a 1,5 mbd durante el mismo período [4].

La caída de los precios mundiales del petróleo, la falta de inversión y por ende el declive del mantenimiento de los campos convencionales, la agresiva política de sanciones y bloqueo económico y comercial por parte de Estados Unidos como medida de presión externa contra el actual gobierno de Nicolás Maduro, así como la fuga de talentos, han golpeado a la industria petrolera venezolana, otrora referente mundial.

PDVSA sufrió una transformación radical de su esencia como corporacióno entidad comercial al servicio del Estado venezolano. Las políticas adoptadas desde 1999 respecto a PDVSA, entre ellas la disminución de los gastos de inversión a través del acceso directo a los recursos financieros de la empresa estatal para ponerlos al servicio del proyecto político, en este caso destinado a los planes de asistencia social y que benefeciaron a la población venezolana más vulnerable, afectaron, del mismo modo, la inversión y mantenimiento eficiente de la compañía, que, sumado a la sanciones y bloqueo económico impuesto por los Estados Unidos, ha traído como consecuencia una disminución en la producción de petróleo, impactando por consiguiente en las exportaciones de este rubro, principal fuente de divisas del país. 
En Venezuela hay una estrecha relación entre el nivel de inversión y de producción. En particular, la caída de la inversión por debajo del nivel necesario para mantener la capacidad se traduce inmediatamente en la caída de la producción".

Las sanciones del año 2019 contra PDVSA aislaron al país de su mayor mercado petrolero -Estados Unidos- quien en 2018 había comprado el 35,6\% de las exportaciones de petróleo de Venezuela. Tal y como se muestra en el siguiente gráfico y tablas de datos correspondientes, durante el 28 de febrero y 01 de abril de 2019, las exportaciones de petróleo venezolano hacia EE.UU. cayeron en un $100 \%$ por primera vez en toda la historia del comercio internacional de Venezuela; las exportaciones a China cayeron en un 49,43\%, debido al rechazo que manifestó el país asiático en comprar el crudo venezolano por las amanezas que recibió de EE.UU, prohibiéndole comerciar con el crudo venezolano. Como consecuencia de esta situación, Venezuela tuvo que reubicar el crudo hacia la India, aumentando de esta manera la cuota para este país en $21,71 \%$ y las de otros países en 1600\% [5] (Ver Figura 1 y Cuadro 1).

Esta caída permaneció durante dos semanas antes de que hubiera un repunte a 139000 barriles por día, y luego una caída hasta los 71000 bpd. Esta situación devastó la producción del petróleo, representando una caída del $36.4 \%$, sólo motivada en este caso por las sanciones de enero de 2019 contra PDVSA [6].

* El aumento de la producción de PDVSA entre 1990 y 1997 tuvo como contraparte la duplicación de la inversión entre estos años. Por el contrario, la caída de la producción hasta 2003, con la excepción de 2001, se debió a la reducción a la mitad de la inversión. Esta información puede ser ampliada en la Revista Venezolana de Economía y Ciencias Sociales v.12 n.1 Caracas abr. 2006. 
Dependencia de la renta petrolera y potencial exportador para la diversificación económica de Venezuela

Figura 1

Exportación de crudo venezolano por país: EE.UU., China, India, otros

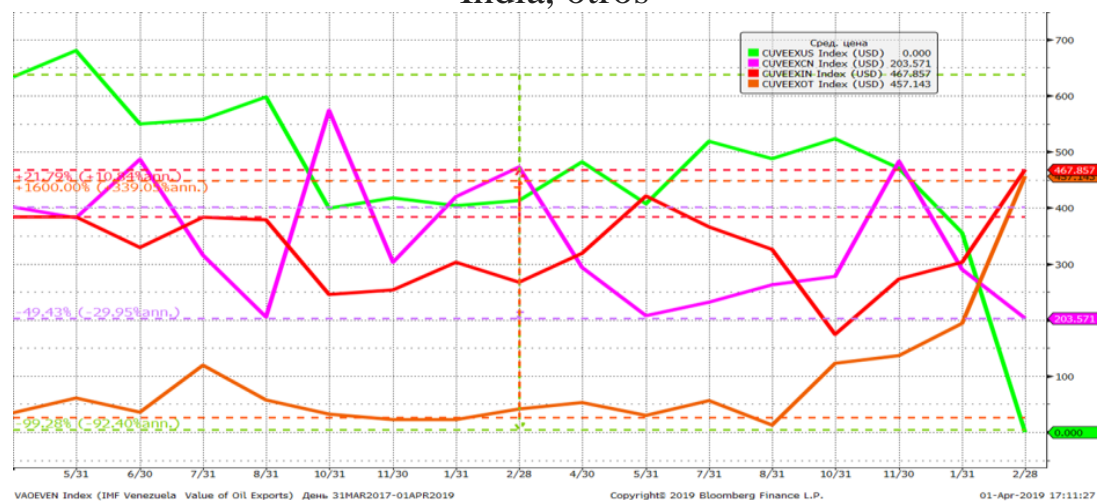

Fuente: elaboración propia según datos de Bloomberg 2019 [5].

Cuadro 1

Exportación de crudo venezolano por país: EE.UU., China, India, otros

\begin{tabular}{|l|c|c|}
\hline Nombre del índice & $\begin{array}{c}\text { Exportación de } \\
\text { crudo al país: }\end{array}$ & Unidades \\
\hline CUVEEXUS Index & EE.UU & 1000 barriles por día \\
\hline CUVEEXCN Index & CHINA & 1000 barriles por día \\
\hline CUVEEXIN Index & INDIA & 1000 barriles por día \\
\hline CUVEEXOT Index & Otras direcciones & 1000 barriles por día \\
\hline
\end{tabular}


Kleidy Y. Espinoza M., Tatiana V. Sidorenko

\begin{tabular}{|c|c|c|c|c|}
\hline Fecha & $\begin{array}{c}\text { CUVEEXUS } \\
\text { Index (USD) }\end{array}$ & $\begin{array}{c}\text { CUVEEXCN } \\
\text { Index (USD) }\end{array}$ & $\begin{array}{c}\text { CUVEEXIN } \\
\text { Index (USD) }\end{array}$ & $\begin{array}{c}\text { CUVEEXOT } \\
\text { Index (USD) }\end{array}$ \\
\hline 28.02 .2019 & 0,000 & 203,571 & 467,857 & 457,143 \\
\hline 31.01 .2019 & 355,775 & 290,323 & 303,226 & 193,815 \\
\hline 30.11 .2018 & 470,797 & 483,333 & 273,333 & 136,667 \\
\hline 31.10 .2018 & 523,365 & 277,419 & 174,194 & 122,903 \\
\hline 31.08 .2018 & 487,178 & 262,903 & 325,806 & 12,581 \\
\hline 31.07 .2018 & 519,054 & 232,258 & 365,468 & 56,452 \\
\hline 31.05 .2018 & 407,851 & 207,613 & 421,355 & 29,677 \\
\hline 30.04 .2018 & 482,218 & 293,333 & 318,862 & 53,333 \\
\hline 28.02 .2018 & 413,413 & 473,214 & 267,857 & 41,429 \\
\hline 31.01 .2018 & 403,658 & 419,355 & 303,226 & 22,581 \\
\hline 30.11 .2017 & 417,375 & 303,333 & 253,333 & 23,333 \\
\hline 31.10 .2017 & 399,082 & 574,194 & 245,520 & 32,258 \\
\hline 31.08 .2017 & 597,534 & 205,161 & 378,914 & 57,287 \\
\hline 31.07 .2017 & 557,278 & 315,161 & 383,452 & 119,355 \\
\hline 30.06 .2017 & 549,726 & 486,667 & 328,761 & 35,000 \\
\hline 31.05 .2017 & 680,349 & 382,258 & 383,820 & 61,290 \\
\hline 31.03 .2017 & 633,830 & 401,613 & 384,743 & 33,871 \\
\hline
\end{tabular}

Fuente: elaboraciónpropia según datos de Bloomberg 2019 [5].

En el ámbito productivo el efecto de arrastre se genera mediante la demanda de bienes y servicios para sus operaciones diarias y proyectos de inversión, incluyendo en este ámbito productivo la producción de insumos y bienes finales para el consumo de las industrias y los hogares (lubricantes, combustibles y aditivos, entre otros). A estos efectos se suman los producidos por el financiamiento del gasto fiscal vía impuestos, tributos y contribuciones fiscales y parafiscales que se derivan de la extracción y el procesamiento de hidrocarburos; la dimensión rentística de la actividad petrolera. 
Dependencia de la renta petrolera y potencial exportador para la diversificación económica de Venezuela

Esta situación es una de las tantas aristas que ha provocado la recesión económica que atraviesa Venezuela y que se ha constituido como la más larga, en comparación con la de los años 1983(viernes negro), 1989 (el caracazo), 1996 (crisis bancaria), 2002 (paro nacional) y 2008 (crisis financiera). Las carcacterísticas que las distingue de la recesión económica actual es que se prolongaron por 2 años, mientras que este año 2021 suma el séptimo año consecutivo de contracción económica [7, p.33].

\section{Políticas comerciales para el desarrollo económico de Venezuela en el siglo XXI}

A partir del año 2001 comienzan a establecerse una serie de objetivos y planes de desarrollo económico para la nación y de comercio y política exterior, como lo son, las Líneas Generales del Plan de Desarrollo Económico y Social de la Nación 20012007, el Primer Plan Socialista de la Nación 2007-2013 y el Segundo Plan Socialista de la Nación 2013-2019.

Dentro de los objetivos históricos nacionales, destaca la prioridad hacia la soberanía alimentaria y lo atinente a la promoción del comercio de exportación agrícola. Nace una vez más la necesidad de buscar mecanismos para la diversificación de las exportaciones. Se incorporan políticas comerciales para implementar el plan nacional de desarrollo agrícola, que contemplan la formación de precios rentables, cupos de importación, disposiciones fiscales y pararancelarias financieras para garantizarar la recuperación de la agricultura nacional.

Asimimo, el Gobierno Bolivariano de Venezuela creó en el año 2005 el Fondo De Desarrollo Nacional (FONDEN), con el objetivo de promover y financiarproyectos de inversión real 
productiva, sociales y comunitarios,y apalancar el crecimiento económico y el desarrollo sostenible del Estado venezolano.

Posteriormente, en el año 2014 anuncia la activación de las Zonas Económicas Especiales (ZEE), representadas en espacios geográficos para el desarrollo productivo de acuerdo con las potencialidades de cada región específicamente. De esta manera se plantea reorganizar la economía con la participación de todos los sectores productivos, tanto privados como públicos, y la captación de la inversión extranjera garantizando la creación de aduanas específicas, así como la liberación de restricciones arancelarias a la importación de materias primas, insumos y maquinarias, con el fin deelevar la capacidad exportadora de Venezuela mediante diversos mecanismos como el Mercado Común del Sur (MERCOSUR).

A finales del 2015, preparó lo que se llama "La Agenda Económica Bolivariana" compuesta de 14 motores de desarrollo productivo nacional. Dentro de este marco se inicia un plan agresivo de llamado a las inversiones foráneas, a países como China, Rusia, República del Congo, Arabia Saudita, Sudáfrica, Canadá, Inglaterra, región de Palestina, Alemania y Suiza, para proyectos que se inscriben en el octavo motor Minero de la Agenda Económica Bolivariana. Se promulga el 24 de febrero del 2016, el Decreto ley que crea la Zona de Desarrollo Estratégico Nacional llamado Arco Minero del Orinoco, que contempla el aprovechamiento de los recursos minerales que posee esta área de $111.800 \mathrm{~km}$ de minerales como oro, hierro, bauxita, coltán, diamante, manganeso y granito.

Pero al igual que en la década de los 60 , a pesar de todas estas políticas en materia económica, son muy pocos los resultados provechosos en términos de transformación estructural, estabilización macroeconómica y del mejoramiento 
Dependencia de la renta petrolera y potencial exportador para la diversificación económica de Venezuela de los niveles de desarrollo del país, debido internamente a la prevalencia de la cultura rentística y deimportaciones, al despliegue de una política expansiva del gasto en lugar de inversión para el desarrollo del aparato productivo,es decir, el uso poco estratégico de los recursos provenientes de la renta petrolera en períodos de bonanzas (entre 2003 y 2013), y externamente debido a la agresiva política de sanciones económicas que ha impuesto los EE.UU contra la economía nacional.

Todo ello se visualiza en la disminución de las exportaciones no tradicionales durante el período 2003-2018. La participación de los bienes industriales no tradicionales en todos los suministros externos disminuyó durante este período casi en un $50 \%$, pasando de $13,0 \%$ a $7,6 \%$. Por su parte, hubo un pequeño aumento de la participación total de las exportaciones agrícolas en todos los suministros externos pasando de $0,2 \%$ a $1,6 \%$. En particular, la participación total del café y del cacao aumentó, aunque también en porcentaje pequeño, pasando del $0,1 \%$ al $0,3 \%[8]$.

El apalancamiento, sin lugar a dudas, del desarrollo de la economía venezolana, viene dada desde décadas de la extracción, producción, exportación del petróleo, por lo que es de suma importancia y necesidad restablecer todo el proceso que implica la recuperación y aumento de la venta del petróleo venezolano en el mercado mundial, tomando en cuenta que incluso sin sanciones económicas, hay muchas dificultades por los altísimos costos de producción del crudo extrapesado del país y que debido a ello es menester aperturar las puertas al mercado internacional y empresas privadas, ya que la industria nacional PDVSA no cuenta en estos momentos con la tecnología, ni los recursos humanos ni financieros, para tal fin. 
Asimismo es menester proporcionar las condiciones políticas que permitan generar confianza en el mercado internacional y financiamiento.

\section{Limitaciones para elcrecimiento de las exportaciones no petroleras de Venezuela en las condiciones de las sanciones económicas occidentales}

Venezuela ha sido objeto de una agresiva y sistemática política de sanciones y bloqueo económico y comercial por parte de los EE.UU. y sus aliados. Desde el año 2014 hasta la actualidad ha recibido más de 150 medidas coercitivas unilaterales [9], es decir, sin que se haya emitido ningún tipo de resolución de la ONU al respecto, que es el organismo internacional facultado para imponer sanciones.

Las actividades de las instituciones financieras norteamericanas en materia de las inversiones en bonos venezolanos fueron bloqueadas a partir de agosto de 2017. PDVSA, que es la principal fuente deingresos y divisas para el país, fue sancionada. Se restringió arbitrariamente el acceso al mercado principal de las ventas. En 2018 Donald Trump prohibió a los ciudadanos estadounidenses realizar operaciones de todo tipo con los títulos de valor de Venezuela, y la adquisición de la nueva criptodivisa PETRO, una vez puesta en circulación por el presidente Nicolás Maduro. Asimismo, entre 2018 y 2019 fueron impuestas sanciones contra el sector bancario venezolano, impidiendo al Estado venezolano la posibilidad de ajustar debidamente cuentas con sus socios comerciales por las importaciones. En 2019 fueron bloqueados los activos de PDVSA por el monto de US\$7 mil millones. Además, quedaron suspendidos los suministros de insumos y repuestos para el mantenimiento del sector petrolero, diluyentes 
Dependencia de la renta petrolera y potencial exportador para la diversificación económica de Venezuela para el petróleo, éste último un factor que también causó la caída del nivel de la extracción y refinación de petroleo [10].

Sin duda, la introducción de sanciones económicas por parte de Estados Unidos y otros países occidentales contra Venezuela puede verse como un incentivo para cambiar el modelo de desarrollo económico nacional, el cual se puede basar en la implementación de una política de sustitución de importaciones con su posterior orientación haciala exportación. Para estos objetivos, la experiencia de Irán y Rusia en la implementación de la política de sustitución de importaciones parece ser la más útil. Lo anterior está determinado por tales factores como la fuerte dependencia de estos países de los ingresosprovenientes de las exportaciones del petróleo y la presión de las sanciones occidentales sobre sus economías.

Sin embargo, como lo demuestra la experiencia mundial, la orientación del desarrollo económico exclusivamentea la sustitución de importaciones puede conllevar enormes riesgos para la economía del país, incluidos los asociados a la producción de productos no competitivos. Como consecuencia, parece que es necesario no sólo utilizar los instrumentos de estimulación estatal del desarrollo de determinadossectoresde la economía nacional, sinoal mismo tiempo desarrollar una estrategia de promoción de bienes a los mercados internacionales. En las condiciones modernas, la política de exportación se convierteen una palanca importante del desarrollo de la producción nacional.

En el contexto de las sanciones económicas de los países occidentales y la situación económica difícil de Venezuela, las posibilidades de introducción de diversos instrumentos para estimular las exportaciones nacionales no petroleras y el 
Kleidy Y. Espinoza M., Tatiana V. Sidorenko

desarrollo del turismo internacional enfrentanciertas limitaciones.

En primer lugar, en las condiciones de las sanciones y la escasez de divisas internacionales, se vuelve problemático adquirir equipos y tecnologías modernas que son necesarios para la organizacion de la producción nacional orientadaal mercado exterior.

En segundo lugar, las posibilidades materiales del gobierno venezolano para financiar industrias promisorias en términos de aumento de los ingresos por exportaciones son limitadas y el interés de la empresa privada es bajo debido a la inestable situación sociopolítica y económica del país y como resultado de la incertidumbre y los riesgos que existen para el desarrollo del emprendimiento nacional.

En tercer lugar, la difícil situación socioeconómica de Venezuela reduce el atractivo inversor de su economía para los inversores extranjeros lo cual lleva a la disminución de la afluencia de inversión extranjera directa (IED) a la economía nacional. Según datos de la Comisión Económica de las Naciones Unidas para América Latina y el Caribe (CEPAL), en el período de 2005 a 2009, en promedio, la IED realizada en la economía venezolana fue de \$ 1403 millones por año, en 2012 alcanzó su máximo histórico de $\$ 5973$ millones [11, p.150]. Sin embargo, de acuerdo con la UNCTAD, la misma bajó a \$ 934 millones en 2019 [12, p. 240].

Como lo evidencian los datos del Informe de Inversión Extranjera Directa en América Latina y el Caribe 2019 elaborado por la Comisión Económica de las Naciones Unidas para América Latina y el Caribe, a pesar del enorme potencial para el desarrollo de la agricultura en Venezuela, en este país no se registraron inversiones de capital extranjero en el sector 
Dependencia de la renta petrolera y potencial exportador para la diversificación económica de Venezuela agroindustrial. Es interesante mencionar que entre 2012 y 2017 casi la mitad de toda la IED realizada en el complejo agroindustrial de la región latinoamericana se dirigió a Brasil (48\%), México (37\%) y Argentina (9\%) [13, p. 141].

En cuarto lugar, la aplicacion de sanciones económicas contra Venezuela ha provocado un deterioro de la imagen del país en el mundo, lo que a su vez también dificulta el desarrollo de contactos con socios extranjeros $\mathrm{y}$, por lo tanto, limita la capacidad del gobierno para diversificar la economía nacional.

En quinto lugar, el temor de los socios extranjeros de Venezuela de caer bajo las sanciones de los Estados Unidos también limita la capacidad del país para implementar políticas destinadas a la diversificacion de la economía nacional.

Sin embargo, a pesar de tantas dificultades con las que se enfrenta el Gobierno venezolano para poder minimizar las consecuencias negativas de las sanciones económicas aplicadas por parte de los países occidentales, es necesario tener presente el hecho de que la economía de ese país sudamericano tiene un grado notable de resistencia económica gracias a su rico potencial de recursos naturales y a las estrategias del Gobierno para desarrollar los mecanismos y políticas de apalancamiento aplicadas recientemente. En ese sentido, el impulso de Irán de aumentar los ingresos por exportaciones no petroleras junto con su mayor dependencia del sector privado podría ser un modelo a seguir.

En la coyuntura actual la devaluación de la moneda nacional de Venezuela podría servir para que sus exportaciones no petroleras fueran más competitivas en los mercados internacionales. Por otra parte, las remesas de los venezolanos, que actualmente representan una de las fuentes importantes de divisas, contribuyen al aumento de las importaciones, y de esa 
Kleidy Y. Espinoza M., Tatiana V. Sidorenko

manera permiten satisfacer las necesidades más prioritarias de la población.

\section{Sectores de la economía nacional venezolana que pueden ser competitivos y con perspectivas en el mercado global}

En el marco de la disminución de la dependenciapetrolera y la diversificación de las exportaciones es necesaria la recuperación socioeconómica de Venezuelay la construcción de un nuevo modelo económico productivo nacional a través del financiamiento e inversión nacional e internacional y del desarrollo de un cuerpo depolíticas para el aprovechamiento de todos los sectores favorables, con ventajas competitivasque se pueden impulsar, como por ejemplo el rescate y expansión del sector siderurgia, petroquímico, turismo, agricultura y así permitir, además, que el país pueda enfrentar los desafíos de la globalización, la integración económica regional y las nuevas tecnologías en los flujos de inversión y comercio mundial.

Por ejemplo, la región de Guayana cuenta con los recursos ideales para el desarrollo del sector metalurgia, como lo son la energía hidroeléctrica, los yacimientos de minerales y la navegación fluvial por el río Orinoco que da acceso a los océanos para el transporte marítimo, aspecto determinante para las exportaciones. En consecuencia, siguen existiendo ventajas comparativas en dicha zona que han facilitado la concentración territorial de las industrias metalúrgicas, conformando de manera natural un "clúster industrial".

De acuerdo con un artículo publicado en la revista SIC de la Fundación Centro Gumilla (junio 22, 2016) de la periodista Clavel Rangel Jiménez, se indica lo siguiente: "Hasta hace una 
Dependencia de la renta petrolera y potencial exportador para la diversificación económica de Venezuela década las empresas básicas representaban el $40 \%$ del PIB no petrolero en el país, y un $10 \%$ del PIB $^{*}$, lo cual proyectaba a la región - junto al desarrollo de la Faja Petrolífera del Orinoco como el colchón de la economía". Además, en las exportaciones no petroleras del año 2003 representaron un 7,5\% de las exportaciones totales del país. Debido a todos estos factores mencionados, la metalurgia es un sector de gran relevancia a considerar para restructurar e impulsar la economía nacional.

Venezuela cuenta con una amplia diversidad geográfica y agro-ecológica, por su condición de país andino, caribeño, llanero y amazónico. Además de poseer las más abundantes reservas de petróleo y gas natural del hemisferio occidental, lo mismo que importantes yacimientos minerales, recursos pesqueros y fuentes hidroeléctricas. Posee unos 53 millones de hectáreas aptas para la agricultura, de las cuales sólo se cultivan 1,5 millones de hectáreas.

Uno de los potenciales para la diversificación de la producción nacionaly por consiguiente de las exportaciones, que debe ser considerada como un área de intervención, es la agricultura, tomando en cuenta la variedad de productos que son cultivables y por las grandes dimensiones de hectáreas que existen para su desarrollo. Entre los principales productos destacan: el café, cacao, la caña de azúcar, tabaco, algodón,

* Con referencia al PIB del Sector Metalurgia, los reportes disponibles del Instituto Nacional de Estadísticas (INE) y del Banco Central de Venezuela en el período 1997-2013 no lo identifican de manera específica como una clase de actividad económica particular, a diferencia de la actividad de la minería (extracción de hierro y otros minerales). Se incluye la metalurgia dentro del PIB Manufactura y no es posible estimar cuánto representa el Sector de la Metalurgia dentro del PIB Manufactura especificamente ya que está generalizada. 
ajonjolí. En cuanto a cultivos mecanizados se encuentran el maíz, arroz, sorgo, trigo. Así como granos o leguminosas de diferentes variedades y frijoles. En cuanto a los cultivos de horticultura se encuentran el tomate, la cebolla, la yuca, la papa, la piña, cambur (plátano), lechoza (papaya) [14].

Además es necesario cambiar la culura de la exportación sólo de materias primas que fue impuesta en tiempos de la colonización por los españoles. Se debe incentivar a través de inversión nacional y extranjera la industrialización en el país, teniendo como objetivo fundamental el procesamiento de las materias primas que se exportan, para que sean reorientadas en un alto porcentaje al mercado interno de manera que las industrias existentes y las que se creen puedanaprovechar su potencial y transformar esas materias primas en productos elaborados para reorientarlas hacia el mercado exportador.

Es necesario establecer una política de innovación tecnológica, industrial y de atracción de la inversión extranjera, así como el desarrollo eficiente de la infraestructura física del país de acuerdo con cada sector a desarrollar.

En cuanto al turismo, Venezuela posee riquezas y bellezas naturales que se combinan entre selva, llanos, playas y montañas, lo que constituye un gran potencial para el destino turístico creándose las condiciones necesarias en cuanto a infraestructura y seguridad. Este sector puede generar altos niveles de ingresos en divisas para la nación.

La era del petróleo ya está llegando a su fin. La energía renovable (principalmente eólica y solar) se está desarrollando rápidamente en muchos países de la región, principalmente en Brasil y Chile. Tiene como ventajas que permite reducir el volumen de emisiones de $\mathrm{CO}^{2}$ a la atmósfera, proporciona precios más bajos, no requiere inversiones tan grandes como la 
Dependencia de la renta petrolera y potencial exportador para la diversificación económica de Venezuela construcción de una central hidroeléctrica (construcción de presas que daña el ambiente ecológico y conduce al desplazamiento de la población a nuevas áreas), y su potencial energético no disminuye, mientras que las reservas del petróleo, carbón y gas son finitas. El Estado venezolano debería aprovechar este tipo de energía a corto y mediano plazo para favorecer también la economía nacional.

\section{Referencias References Ссылки}

1. Bitar, Sergio. \& Troncoso, Eduardo. Venezuela: hacia una nueva estrategia industrial. Available

at: https://static.nuso.org/media/articles/downloads/990_1.pdf 15.07.2020).

2. Banko, Catalina. Industrialización y Políticas Económicas en Venezuela. Available at: https://core.ac.uk/download/pdf/268360109.pdf (accessed 15.07.2020).

3. Gutiérrez, A. S. El capitalismo rentístico y el sistema alimentario venezolano (SAV). Agroalimentaria, Universidad de Los Andes, Mérida, 2010, num. 30, pp. 129-150.

4. Curcio, Pasqualina. Caída de la producción en Venezuela. Causas. Available https://observatoriodetrabajadores.wordpress.com/2019/10/31/caida-de-laproduccion-en-venezuela-causas pascualina-curcio-curcio/ (accessed 21.08.2020).

5. Exportación de crudo de Venezuela.Bloomberg Finance L.P. Elaboración propia del autor en el Laboratorio de Bloomberg de la Universidad Financiera dependiente del Gobierno de Rusia. Bajo la referencia: Venezuela STOCK MKT INDEX ESCT. CUVEEXUS, Index. (2019).

6. Sanciones económicas como castigo colectivo: El caso de Venezuela. Available at: http://cepr.net/images/stories/reports/venezuelasanctions-2019-05-spn.pdf (accessed 23.10.2020).

7. Key, R. y Villarroel, C. El Petróleo será insuficiente: El colapso de la industria petrolera y la crisis venezolana. DebatesIESA. Caracas, num. 2. 2018, pp. 29-33. 
Kleidy Y. Espinoza M., Tatiana V. Sidorenko

8. Observatorio de Complejidad Económica (OEC). Available at: https://oec.world/es/profile/country/ven/ (accessed 17.01.2021).

9. Medidas coercitivas de EE.UU. contra Venezuela. Available at: https://www.telesurtv.net/news/venezuela-cronologia-medidas-sancioneseeuu-20200213-0004.html. (accessed 17.11.2020).

10. Alekséenko O. A., Pyatakov A. N. Venezuela: prueba por la crisis. Iberoamérica. Moscú, 2019, num. 2 pp. 57-83.

11. La Inversión Extranjera Directa en América Latina y el Caribe 2020. CEPAL, 2020, pp. 141-150.

12. World Investment Report 2020. InternationalProduction beyond the Pandemic. UNCTAD, 2020, 240 p.

13. La Inversión Extranjera Directa en América Latina y el Caribe 2019. CEPAL, 2019, $141 \mathrm{p}$.

14. MSC Invernaderos. Principales cultivos agrícolas en Venezuela. Available at: https://grupomsc.com/blog/agricultura/principalescultivos-devenezuela (accessed 15.08.2020).

\section{Bibliografía Bibliography Библиография}

15. Sanchez, Clara. Venezuela en la geopolítica de los alimentos. Available at:

https://alimentosypoder.com/2019/07/07/delavenezuela-exportadora-decacao-y-cafe-al petroleo/\#_ftn23 (accessed 12.08.2020).

16. Rodríguez, P.L. y Rodríguez, L.R. El petróleo como instrumento de progreso. Ediciones IESA. Caracas, 2013, num. 2, 244 p.

17. Asociación Latinoamericana de Libre Comercio. Available at: https://www.ecured.cu/Asociaci\%C3\%B3n_Latino\%C2\%ADamericana_de_ Libre_Comercio(accessed 20.10.2020).

18. La Incorporación de Venezuela al Acuerdo Subregional de Integración Andino. Available at: https://www.repository.fedesarrollo.org.co/bitstream/handle/11445/2810/Co_ Eco_Abril_1973_Fedesarrollo.pdf?sequence=2\&isAllowed=y (accessed 20.07.2020).

19. Izarra A., Boscán A.L. Cronología de una implosión. La década final de IV República. Minci. Caracas, 2011, num. 2, pp. 1-173.

20. Rodríguez, Margarita. La fatalista "profecía" sobre Venezuela que hizo un joven hace más de 80 años. Available at: https://www.bbc.com/mundo/noticias-53082364 (accessed 15.07.2020). 
Dependencia de la renta petrolera y potencial exportador para la diversificación económica de Venezuela

21. Negocios Rentables. Cuáles son los productos que más exporta Venezuela. Available at: https://negociosrentablesfx.com/paises/cuales-losproductos-mas-exporta-venezuela/ (accessed 15.07.2020).

22. Expansión Datosmacro.com. Precio del petróleo OPEP por barril. Available at: https://datosmacro.expansion.com/materiasprimas/opec?dr=2019-01 (accessed 18.07.2020).

23. Gobierno Bolivariano de Venezuela, Ministerio del Poder Populara de Economía y Finanzas. Fondo De Desarrollo Nacional SA. (FONDEN). Available at: http://www.mppef.gob.ve/fondo-nacional-para-el-desarrolloendogeno-fonden/ (accessed 13.08.2020).

24. República Bolivariana de Venezuela, Ministerio del Poder Popular para la Planificación. Zonas Económicas Especiales. Available at: https://issuu.com/productorabacoa/docs/librillozonaseconomicasespeciales (accessed 13.08.2020).

25. Gaceta Oficial №40.855, Decreto No. 2.248, con Rango Valor y Fuerza de Ley Orgánica, de la República Bolivariana de Venezuela, 2016.

26. Statista. Evolución anual del precio medio del petróleo crudo fijado por la Organización de Países Exportadores de Petróleo (OPEP) de 1960 a 2019. Available at: https://es.statista.com/estadisticas/635114/preciomediodel-crudo-fijado-por-la-opep/(accessed 14.08.2020).

27. García, Arnoldo. Exportaciones no tradicionales se han duplicado en lo que va de 2017. Available at: https://www.finanzasdigital.com/2017/03/arnoldo-garcia-exportaciones-notradicionales-se-han-duplicado-en-lo-que-va-de-2017/ (accessed 14.08.2020).

28. El Convenio Cambiario $\mathrm{N}^{\circ} 34$. Gaceta Oficial $\mathrm{N}^{\circ} 41.102$ de fecha originaria 11.02.2016.

29. Vida Agro. Venezuela posee más de 50 millones de hectáreas con potencial agrícola. Available at: http://www.vidaagro.com.ve/venezuelaposee-mas-de-50-millones-de-hectareas-con-potencial-

agricola/\#: :text=De\%20los\%2091\%20millones\%20de,es\%20apta\%20para\% 20la\%20agricultura (accessed 15.08.2020).

30. Castillo, Julián. ¿Cómo ha sido la caída de la producción agropecuaria en los últimos 20 años? Available at: //eldiario.com/2019/07/29/como-ha-sido-la-caida-de-la-produccionagropecuaria-en-los-ultimos-20-anos/ (accessed 15.08.2020). 